\title{
A Generalized Constitutive Equation with Distributed Order Derivative for Viscoelastic Solid
}

\author{
Lingjie Duan and Junsheng Duan
}

\begin{abstract}
In order to generalize the Kelvin-Voigt model describing viscoelastic solids, we present a constitutive equation with distributed order derivative. Using the Laplace transform and its numerical inverse, we consider the creep compliance, creep recovery, relaxation modulus, and stress response to harmonic deformation. The results show that the constitutive equation indeed characterizes viscoelastic solids and is a generalization of the Kelvin-Voigt model. Meanwhile, we discuss the effect of the modelling parameter on viscoelasticity.
\end{abstract}

Index Terms-Constitutive equation, distributed order derivative, fractional calculus, response.

\section{INTRODUCTION}

Viscoelasticity is the property of materials that exhibit both viscous and elastic characteristics when undergoing deformation. Many polymers and biological tissues exhibit such a behavior. In recent decades, the viscoelasticity theory has developed considerably partly because of the wide use of polymers in various fields of engineering. Also viscoelastic materials are extensively applied to cushion shock, from running shoes to packing materials.

According to the linear viscoelasticity theory, the viscoelastic body can be considered as a linear system with the stress $\sigma(t)$ (or strain $\varepsilon(t)$ ) as the excitation function (input) and the strain $\varepsilon(t)$ (or stress $\sigma(t))$ as the response function (output). In this respect, the response functions to an excitation expressed by the Heaviside unit step function $\Theta(t)$ are known to play a fundamental role both from a mathematical and physical point of view.

We denote by $J(t)$ the strain response to the unit step of stress and by $G(t)$ the stress response to a unit step of strain. The functions $J(t)$ and $G(t)$ are usually referred to as the creep compliance and relaxation modulus respectively, or, simply, the material functions of the viscoelastic body. In view of the causality requirement, both functions are vanishing for $t<0$. For $0<t<+\infty, J(t)$ is a nondecreasing function and $G(t)$ is a nonincreasing function.

The limiting values of the material functions for $t \rightarrow 0^{+}$ and $t \rightarrow+\infty$ are related to the instantaneous (or glass) and

\footnotetext{
Manuscript received January 8, 2017; revised April 5, 2017. This work was supported in part by the Natural Science Foundation of Shanghai (No.14ZR1440800).

L. Duan is with the State Key Laboratory of Powder Metallurgy, Central South University, Changsha 410083, P.R. China (e-mail: dlj14141144@hotmail.com).

J. Duan is with the School of Sciences, Shanghai Institute of Technology, Shanghai 201418, P.R. China (e-mail: duanjs@sit.edu.cn).
}

equilibrium behaviours of the viscoelastic body, respectively. As a consequence, it is usual to call $J\left(0^{+}\right)$the glass compliance, $J(+\infty)$ the equilibrium compliance, and $G\left(0^{+}\right)$ the glass modulus, $G(+\infty)$ the equilibrium modulus. They satisfy the relations

$$
J\left(0^{+}\right)=1 / G\left(0^{+}\right), J(+\infty)=1 / G(+\infty),
$$

for linear viscoelastic material models [1]-[3]. Generally one may define a fluid if it can creep indefinitely under constant stress, namely when it relaxes to zero under constant deformation. In contrast, viscoelastic solid means its equilibrium compliance is finite, i.e. its equilibrium modulus is nonzero.

Fractional calculus has been applied to mathematical description of real problems arising in different fields of science and engineering, such as viscoelasticity, anomalous diffusion, control theory, etc [2]-[9]. It is capable for describing memory and hereditary properties of various materials and processes. In viscoelasticity theory, fractional calculus has been used to establish constitutive equations conveniently and effectively [2]-[7], [10]-[14].

Let $f(t)$ be piecewise continuous on $(0,+\infty)$ and integrable on any finite subinterval of $(0,+\infty)$. Then the Riemann-Liouville fractional integral of $f(t)$ of order $\beta$ is defined as

$$
{ }_{0} J_{t}^{\beta} f(t)=\int_{0}^{t} \frac{(t-\tau)^{\beta-1}}{\Gamma(\beta)} f(\tau) d \tau, t>0,
$$

where $\beta$ is a positive real number, and $\Gamma(\cdot)$ is Euler's gamma function. For complementarity, we define ${ }_{0} J_{t}^{0} f(t)=f(t)$.

Let $\alpha$ be a positive real number satisfying $m-1<\alpha \leq m$ and $m \in \mathbb{N}^{+}$, where $\mathbb{N}^{+}$is the set of positive integers. Then the Riemann-Liouville fractional derivative of $f(t)$ of order $\alpha$ is defined, when it exists, as

$$
f^{(\alpha)}(t)={ }_{0} D_{t}^{\alpha} f(t)=\frac{d^{m}}{d t^{m}}\left({ }_{0} J_{t}^{m-\alpha} f(t)\right), t>0 .
$$

Scott-Blair [4], [15] proposed a fractional constitutive equation $\sigma(t)=E \varepsilon^{(\alpha)}(t) \quad$, where $E$ and $\alpha$ are material-dependent constants and $0<\alpha<1$, to characterize a viscoelastic material whose mechanical properties are intermediate between those of a pure elastic solid (Hooke model) and a pure viscous fluid (Newton model). In the monographs [1], [2], this relation was called as the Scott-Blair model. In [6], a fractional calculus element whose 
constitutive law obeys stress is proportional to a fractional derivative of strain is said to be a spring-pot.

Different fractional constitutive equations have been proposed, such as the fractional Maxwell, Kelvin-Voigt, and Zener models by replacing Hooke's and Newton's classical elements by the Scott-Blair element [11]-[14], [16], [17].

The Kelvin-Voigt model for viscoelastic body consists of a spring and dashpot in parallel. The constitutive equation reads

$$
\sigma(t)=E \varepsilon(t)+\eta \dot{\varepsilon}(t)
$$

from which the creep compliance and the relaxation modulus are derived

$$
J(t)=\frac{1}{E}\left(1-e^{-E t / \eta}\right),
$$

and

$$
G(t)=E \Theta(t)+\eta \delta(t)
$$

respectively, where $\Theta(t)$ is the Heaviside unit step function and $\delta(t)$ is the Dirac delta function. The finite equilibrium compliance and the nonzero equilibrium modulus

$$
J(+\infty)=\frac{1}{E}, G(+\infty)=E,
$$

mean the viscoelastic body is solid-like.

In [18], a generalized constitutive equation with the distributed order derivative

$$
\sigma(t)=E \int_{0}^{1} \tau^{\alpha} \varepsilon^{(\alpha)}(t) d \alpha,
$$

is introduced. Seemingly, the Hooke model and the Newton model, which are expressed by terms of strain and its derivative respectively, are interpolated on the right hand side of (7), and (7) is a generalization of the Kelvin-Voigt model (4). But the model (7) does not characterize viscoelastic solids since the equilibrium compliance and the equilibrium modulus are proved out to be

$$
J(+\infty)=+\infty, G(+\infty)=0 .
$$

In this article, we propose the constitutive relation with distributed order derivative

$$
\sigma(t)=E \varepsilon(t)+E \int_{0}^{1} \tau^{\alpha} \varepsilon^{(\alpha)}(t) d \alpha .
$$

We show that this constitutive relation characterizes viscoelastic solid and is a real generalization for the Kelvin-Voigt model.

In next section, we investigate the creep compliance, creep-recovery, relaxation modulus, energy dissipation or hysteresis under periodic deformation, etc. by using the constitutive equation (9).

\section{RESPONSE ANALYSIS AND SIMULATION}

We examine the creep compliance, creep recovery, relaxation modulus, and stress response to harmonic deformation for the constitutive relation (9) and consider the effects of the parameter $\tau$ to the material functions.

Applying the Laplace transform to (9) and then integrating with respect to $\alpha$ yield

$$
\bar{\sigma}(s)=\left(E+\frac{E(\tau s-1)}{\ln (\tau s)}\right) \bar{\varepsilon}(s) .
$$

In this article, we use the following definition and notation of the Laplace transform of function $f(t)$

$$
L[f(t)]=\bar{f}(s)=\int_{0^{-}}^{\infty} f(t) e^{-s t} d t
$$

If we take $\sigma(t)=\Theta(t)$, the Laplace transform of creep compliance $J(t)$ is obtained

$$
\bar{J}(s)=\frac{\ln (\tau s)}{E s \ln (\tau s)+E s(\tau s-1)} .
$$

From the initial value and final value theorems, we have

$$
\begin{aligned}
& J\left(0^{+}\right)=\lim _{s \rightarrow \infty} s \bar{J}(s)=0, \\
& J(+\infty)=\lim _{s \rightarrow 0} s \bar{J}(s)=\frac{1}{E} .
\end{aligned}
$$

Note that no instantaneous elastic deformation is possible, but the model undergoes finite creep like the Kelvin-Voigt model. We calculate the inverse Laplace transform of (12) numerically by the algorithm and the MATHEMATICA subroutine presented in [19].

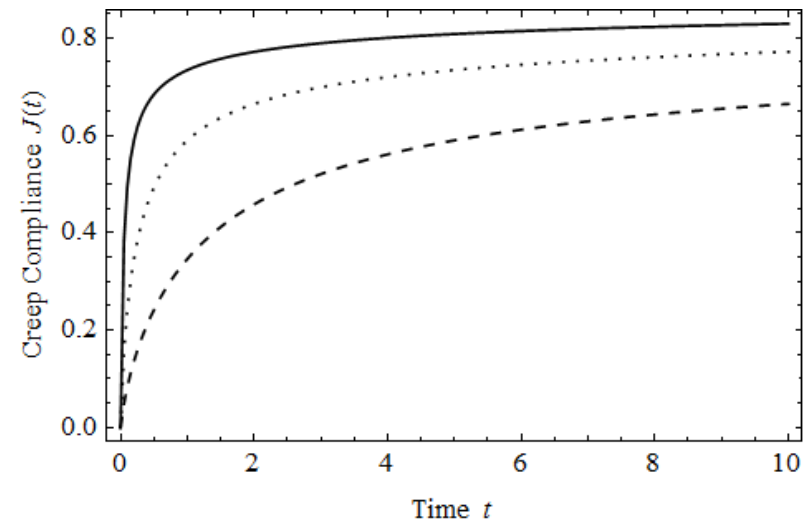

Fig. 1. Curves of creep compliance $J(t)$ for $E=1$ and for $\tau=0.2$ (solid line), $\tau=1$ (dot line) and $\tau=5$ (dash line).

In Fig. 1, we display the curves of creep compliance $J(t)$ for $E=1$ and for $\tau=0.2,1$ and 5. The values of $\tau$ can represent the strengths of elasticity and viscosity of material. For $\tau=0.2$, the model shows stronger elasticity while for $\tau=5$, the stronger viscosity is reflected.

We examine the creep recovery response by removing the load suddenly. Thus the applied stress can be expressed as $\sigma(t)=\Theta(t)-\Theta(t-T)$. Its Laplace transform is $\bar{\sigma}(s)=\frac{1}{s}\left(1-e^{-T s}\right)$. We obtain the Laplace transform of creep recovery response

$$
\bar{\varepsilon}(s)=\frac{\ln (\tau s)\left(1-e^{-T s}\right)}{E s \ln (\tau s)+E s(\tau s-1)} .
$$

In fact, the creep recovery response is

$$
\varepsilon(t)=J(t)-J(t-T) \Theta(t-T),
$$

where the final value is $\varepsilon(+\infty)=0$. The model exhibits an 
eventually complete creep recovery. In Fig. 2, we display the curves of creep recovery response $\varepsilon(t)$ for $E=1, T=4$ and for $\tau=0.2,1$ and 5 .

Next, we examine the relaxation modulus by taking the unit step strain $\varepsilon(t)=\Theta(t)$. The Laplace transform of relaxation modulus $G(t)$ takes the form

$$
\bar{G}(s)=\frac{E}{s}+\frac{E(\tau s-1)}{s \ln (\tau s)} .
$$

The initial value and final value of relaxation modulus $G(t)$ are given as

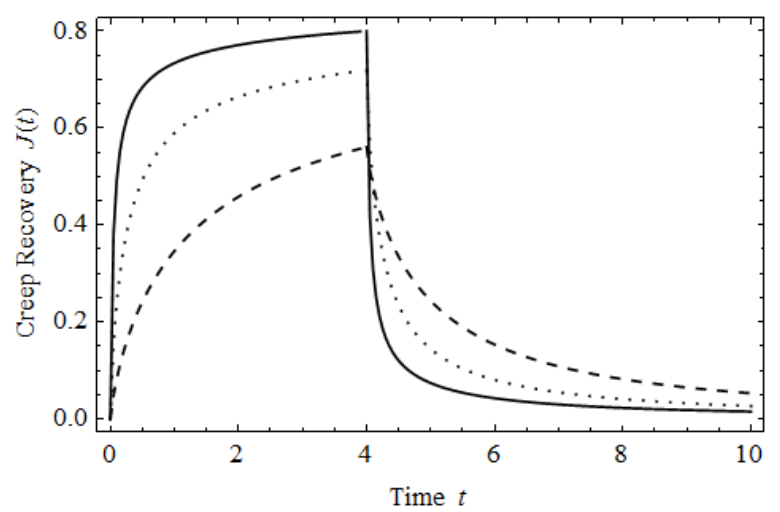

Fig. 2. Creep recovery responses $\varepsilon(t)$ for $E=1, T=4$ and for $\tau=0.2$ (solid line), $\tau=1$ (dot line) and $\tau=5$ (dash line).

$$
\begin{aligned}
& G\left(0^{+}\right)=\lim _{s \rightarrow \infty} s \bar{G}(s)=+\infty, \\
& G(+\infty)=\lim _{s \rightarrow 0} s \bar{G}(s)=E .
\end{aligned}
$$

The model affords a nonzero equilibrium modulus like the Kelvin-Voigt model and presents equilibrium elasticity. In Fig. 3, We plot the curves of the relaxation modulus $G(t)$ versus $t$ for $E=1$ and for $\tau=0.2,1$ and 5. For large value $\tau=5$, stress relaxes slower, which means stronger viscosity.

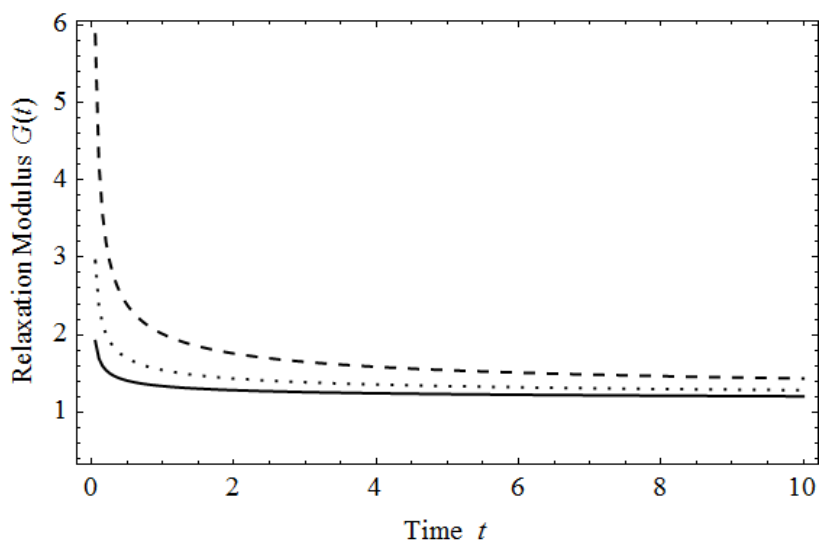

Fig. 3. Curves of the relaxation modulus $G(t)$ for $E=1$ and for $\tau=0.2$ (solid line), $\tau=1$ (dot line) and $\tau=5$ (dash line).

Finally, we investigate the stress response to harmonic strain $\varepsilon(t)=\sin (\omega t)$. The Laplace transform of stress response is

$$
\bar{\sigma}(s)=\left(E+\frac{E(\tau s-1)}{\ln (\tau s)}\right) \frac{\omega}{s^{2}+\omega^{2}} .
$$

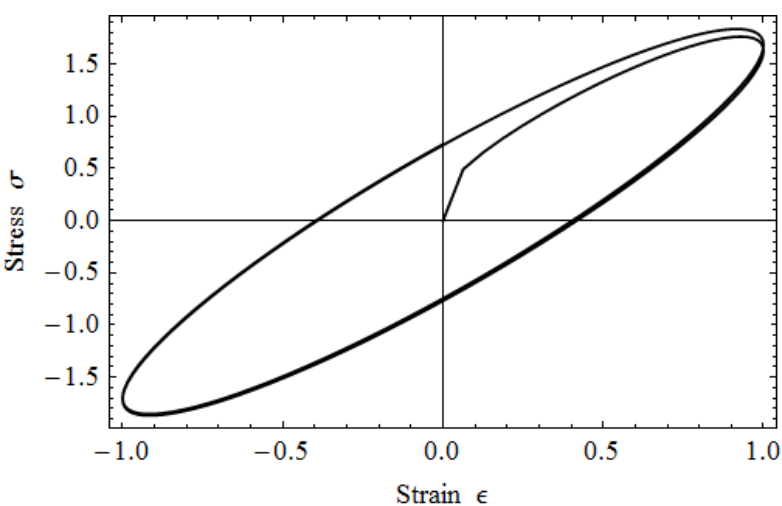

Fig. 4. Stress-strain hysteresis loop for $E=1, \omega=2 \pi$ and $\omega=2 \pi$.

In Figs. 4-6, stress-strain hysteresis loops are shown for $E=1, \omega=2 \pi$ and for $\tau=0.2,1$ and 5, respectively. Phase difference between stress and strain is obvious, which means hysteresis and energy dissipation. The three figures display that for the three values of $\tau$, as $\tau$ increases, more energy is dissipated and so viscosity becomes stronger.

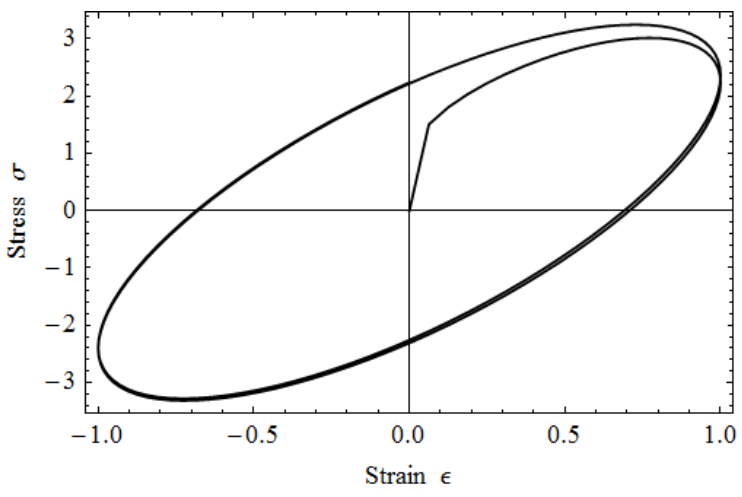

Fig. 5. Stress-strain hysteresis loop for $E=1, \omega=2 \pi$ and $\tau=1$.

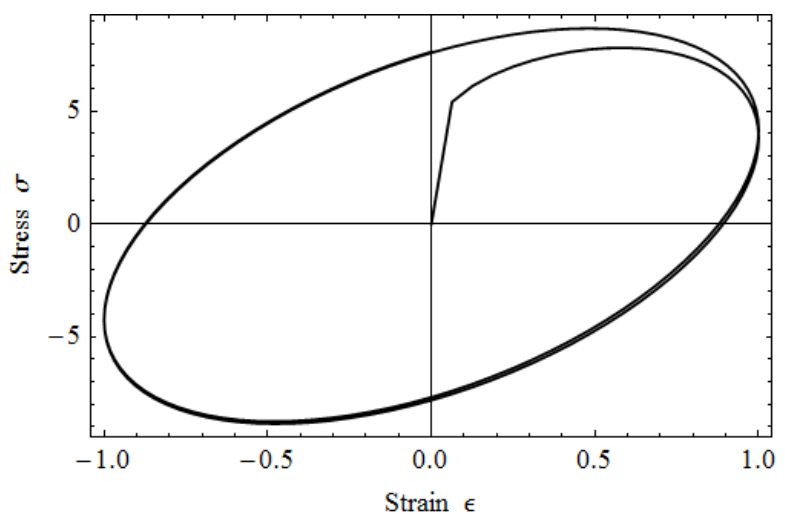

Fig. 6. Stress-strain hysteresis loop for $E=1, \omega=2 \pi$ and $\tau=5$.

We indicate that we examined the equation

$$
\sigma(t)=E \varepsilon(t)+\eta \dot{\varepsilon}(t)+E \int_{0}^{1} \tau^{\alpha} \varepsilon^{(\alpha)}(t) d \alpha,
$$

but in this model, the creep compliance and the relaxation modulus have the same initial values and final values as that in model (9). So model (9) is enough for our aim.

\section{CONCLUSION}

In this article, we present and investigate a generalized constitutive equation with distributed order derivative 


$$
\sigma(t)=E \varepsilon(t)+E \int_{0}^{1} \tau^{\alpha} \varepsilon^{(\alpha)}(t) d \alpha .
$$

Using the Laplace transform and its numerical inverse, we consider the creep compliance, creep recovery, relaxation modulus, and stress response to harmonic deformation. The results show that the constitutive equation characterizes viscoelastic solids and is a generalization of the Kelvin-Voigt model. The effect of the parameter $\tau$ on viscoelasticity is displayed. All the figures are generated by using MATHEMATICA 8.

\section{REFERENCES}

[1] D. R. Bland, The Theory of Linear Viscoelasticity, Oxford, U.K.: Pergamon, 1960, ch. 3.

[2] F. Mainardi, Fractional Calculus and Waves in Linear Viscoelasticity, London, U.K.: Imperial College, 2010, ch. 3.

[3] F. Mainardi and G. Spada, "Creep, relaxation and viscosity properties for basic fractional models in rheology,” Eur. Phys. J. Special Topics, vol. 193, no. 1, pp. 133-160, March 2011.

[4] G. W. Scott-Blair, Survey of General and Applied Rheology, London, U.K.: Pitman, 1949, ch 4.

[5] A. N. Gerasimov, "A generalization of linear laws of deformation and its application to inner friction problems," Prikl. Mat. Mekh., vol. 12, pp. 251-259, 1948.

[6] R. C. Koeller, "Applications of fractional calculus to the theory of viscoelasticity,” J. Appl. Mech., vol. 51, pp. 299-307, June 1984.

[7] I. Podlubny, Fractional Differential Equations, San Diego, CA: Academic, 1999, ch. 10.

[8] Y. Chen and K. L. Moore, "Analytical stability bound for a class of delayed fractional-order dynamic systems,” Nonlinear Dynam., vol. 29 no. 1, pp. 191-200, July 2002.

[9] W. Chen, "An intuitive study of fractional derivative modeling and fractional quantum in soft matter," J. Vib. Control, vol. 14, no. 9, pp. 1651-1657, September 2008.

[10] M. Caputo and F. Mainardi, "Linear models of dissipation in anelastic solids," La Rivista del Nuovo Cimento, vol. 1, no. 2, pp. 161-198, April 1971.

[11] L. Rogers, "Operators and fractiorial derivatives for viscoelastic constituitive equations,” J. Rheol., vol. 27, no. 4, pp. 351-372, August 1983.

[12] R. L. Bagley and P. J. Torvik, "On the fractional calculus model of viscoelastic behavior,” J. Rheol., vol. 30, no. 1, pp. 133-155, February 1986.

[13] H. Schiessel, R. Metzler, A. Blumen, and T. F. Nonnenmacher, "Generalized viscoelastic models: their fractional equations with solutions,” J. Phys. A: Math. Gen., vol. 28, no. 23, pp. 6567-6584, December 1995.

[14] T. Pritz, "Analysis of four-parameter fractional derivative model of real solid materials,” J. Sound Vibr., vol. 195, no. 1, pp. 103-115, August 1996.

[15] G. W. Scott-Blair, "Analytical and integrative aspects of the stress-strain-time problem,” J. Scientific Instruments, vol. 21, no. 5, pp. 80-84, May 1944.

[16] C. Huang and J. S. Duan, "Steady-state response to periodic excitation in fractional vibration system,” J. Mechanics, vol. 32, no. 1, pp. 25-33, February 2016.

[17] J. S. Duan and X. Qiu, "The periodic solution of Stokes' second problem for viscoelastic fluids as characterized by a fractional constitutive equation,” J. Non-Newtonian Fluid Mechanics, vol. 205, pp. 11-15, March 2014.

[18] T. M. Atanackovic, "A generalized model for the uniaxial isothermal deformation of a viscoelastic body," Acta Mechanica, vol. 159, no. 1, pp. 77-86, March 2002.

[19] J. Abate and P. P. Valkó, "Multi-precision laplace transform inversion,” Int. J. Numer. Meth. Engng., vol. 60, no. 5, pp. 979-993, May 2004.

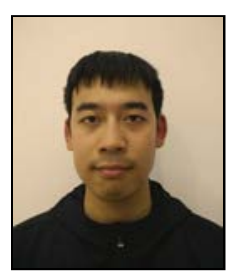

Linjie Duan was born in Huhhot, Inner Mongolia, P.R. China in 1992. He received his bachelor's degree in materials chemistry in 2015 from Central South University, Changsha, P.R. China. Now he is a graduate student for a master's degree in materials engineering at the State Key Laboratory of Powder Metallurgy, Central South University, Changsha, P.R. China, and engages in research work of material mechanics and powder metallurgy materials.

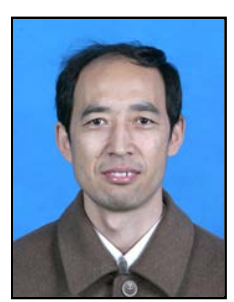

Junsheng Duan was born in Huhhot, Inner Mongolia, PR China in 1965. He received his master's degree in applied mathematics in 1996 from Inner Mongolia University, Huhhot, P.R. China. In 2002, he received his $\mathrm{PhD}$ in applied mathematics from Shandong University, Jinan, P.R. China.

He is a professor at School of Sciences, Shanghai Institute of Technology in Shanghai, P.R. China. Before coming to Shanghai Institute of Technology, he worked at College of Science, Tianjin University of Commerce, Tianjin, P.R. China. His research interests are viscoelastic material mechanics, applied mathematics, and differential equations in mathematical physics. He has published over 90 reviewed papers in mechanics and mathematical physics. 